\title{
DESARROLLO DE UN INDICE BIOLOGICO PARA ESTIMAR LA CALIDAD DE LAS AGUAS DE LA CUENCA DEL DUERO
}

\author{
M. González del Tanago (1) y D. García Jalón (2) \\ Departamento de Hidrología (1); Departamento de Zoología (2). E.T.S.Ingeniero de Montes. Madrid. Universidad Poli- \\ técnica de Madrid.
}

Palabras Clave: Water quality, Duero river (Spain)

\begin{abstract}
BIOLOGICAL INDEX FOR ESTIMATING WATER QUALITY IN THE DUERO BASIN

In this paper, an index for estimating biological quality of water, adapted to Duero Basin fauna, is elaborated. This index takes into account the interference of river zonation with the indicator species concept, together with synecological information.

A comparison of other indices in the same area, evaluating the different results, is also given.
\end{abstract}

\section{INTRODUCCION}

Los índices biológicos se han venido utilizando desde principios de siglo, con muy distintas finalidades. Puede decirse que el primer intento de su aplicación lo hicieron Kolkwitz y Marsson (1902, 1908, 1909) al definir los distintos grados o etapas de recuperación de un río después de haber sufrido una contaminación organica y establecer el conocido sistema de las saprobias.

En Inglaterra y América del Norte se han producido, básicamente, dos tendencias en el desarrollo de los índices biológicos: los índices bióticos y los índices de diversidad.

Entre los primeros cabe citar el de Beck (1955), el de Trent (Woodiwiss, 1964) y el de Chandler (1970). Posteriormente se han desarrollado otras índices más adaptados a las especies existentes en la zona de aplicación, como el de Tuffery y Verneaux (1967). García de Jalón y González del Tanago (en prensa) y otros de carácter general como el utilizado por González del Tanago et. al. (1979).

En general, lo que expresan los índices bióticos es la presencia y abundancia de ciertas "especies indicado- ras" de la calidad de las aguas, ponderando estas últimas según su tolerancia a la contaminación orgánica. Una alternativa al estudio de la presencia de estas "especies indicadoras" para conocer el estado o calidad de las aguas es la utilización de la estructura de la comunidad en términos de su diversidad, es decir, atendiendo a la proporción de individuos de cada especie en la misma.

Entre los índices de diversidad más utilizados cabe citar el de Shannon-Weaver (1963), el de Margalef (1951), el de Menhinick (1964) y el de Simpson (1949).

Todos estos índices biológicos citados han sido objeto de numerosos estudios descriptivos (Wilhm, 1975; Verneaux, 1976; Hellawell, 1978) y críticos, al aplicarse simultáneamente algunos de ellos a una misma zona de estudio (Balloch et al., 1976; Ghetti y Bonazzi, 1977; Jensen y Aagaard, 1981). Su utilidad para estimar la calidad de las aguas ha sido puesta de manifiesto en numerosas ocasiones (Hawkes, 1978; Hellawell, 1978) comprobando, a su vez, el interés de considerar en los mismos tanto el aspecto autoecológico de la comunidad que se pretende valorar a través del índice, como su aspecto sinecológico, relacionado con la abundancia de cada especie en la misma. 


\section{METODOLOGIA}

Se han estudiado las características físico-químicas y biológicas en 76 localidades de la Cuenca del Duero, distribuídas en 22 ríos, cuyos resultados han sido expuestos en otro lugar (González del Tánago y García de Jalón, en prensa).

Con el fin de estimar la calidad biológica de las aguas de la zona estudiada, en estrecha relacióncon su calidad físico-química, hemos establecido un índice o expresión matemática que integra los resultados obtenidos, relativos a las comunidades de macroinvertebrados que viven en los distintos tramos de los ríos.

La mayoría de los índices biológicos que hoy día tienen mayor aceptación están basados en una ponderación de las especies según su tolerancia al grado de contaminación orgánica de las aguas o, dicho de otra forma, según su necesidad de oxígeno disuelto en el medio acuático, resultando que las especies que viven en los tramos altos de los ríos están siempre más ponderadas que las que se sitúan en los tramos bajos, igual que sucede con las especies que viven en facies Iótica, que puntúan más que las que se encuentran en la facies léntica de los ríos. Y esto hace que el valor del índice sea siempre más alto en los tramos de rhithron que en los correspondientes al potamon, aún sin que varíen las condiciones intrínsecas de calidad de las aguas.

Tratando de evitar esta influencia de la zonación natural de los ríos sobre el valor del índice biológico, y teniendo en cuenta las consideraciones anteriormente comentadas respecto a los factores que deben ser integrados en el mismo, hemos establecido la siguiente relación o expresión numérica de la calidad biológica de las aguas:

$$
\mathrm{Ib}=\mathrm{f}_{1}(\mathrm{E}) \times f_{2}(\mathrm{C})
$$

siendo: Ib el valor del índice biológico

$f_{1}$ (E) una función de la estructura de la comunidad, como factor sinecológico de la misma.

f2 (C) una función de la composición de la comunidad, como factor autoecológico de la misma.

El índice propuesto adopta expresiones distintas según el tramo de río que se quiera valorar, al ser diferente, en cada caso, el factor autoecológico del mismo.

Así, la función de la composición de la comunidad toma las siguientes expresiones según el tramo del río considerado:
Zona de Rhithron: $f_{2}(C)=(2 \operatorname{sint}+\operatorname{sind}+2$ seut -5 scont. $)$

Zona de Epipotamon: $f_{2}(C)=(3 \sin t+\operatorname{sind}-1 / 2$ seut $-5 \operatorname{scont})$

Zona de Mesopotamon: $\mathrm{f}_{2}(\mathrm{C})=(4 \operatorname{sint}+\operatorname{sind}-10 \operatorname{scont}) \times 3$

donde se representa el número de especies presentes en la comunidad, distinguiendose entre:

- Especies intolerantes: las que para su existencia necesitan altos contenidos de oxígeno disuelto en las aguas, no tolerando en absoluto la contaminación orgánica de las mismas. Dichas especies son frecuentes, y a menudo abundantes, en los tramos altos de los ríos donde su presencia tiene un carácter "normal" o esperado. Si están también presentes en los tramos más bajos se consideran "fuertemente indicadoras de buena calidad de las aguas", al tener un carácter marcadamente estenoico.

-Especies indiferentes: Especies que pueden vivir en condiciones de las aguas muy variables, estando más ligadas a un microhábitat o tacies concreta el río que a una zona o tramo del mismo. Su presencia y abundancia en las aguas refleja únicamente la existencia de estos microhábitats requeridos por ellas, no teniendo ningún valor indicador de la "calidad de las aguas" al ser especies estenotopas de carácter eurioico.

- Especies de aguas eutróficas: Son las que viven preferentemente en los tramos bajos de los ríos, donde las aguas están de forma natural más eutrofizadas que en los tramos altos. Estas especies pueden existir a lo largo de todo el río pero su abundancia es siempre mucho mayor en la zona de potamon. En estas zonas bajas su presencia es "normal" y esperada, debido al carácter eurioico, y a veces euritopo, de las mismas. Cuando son abundantes en los tramos altos de los ríos denotan una eutrofización de las aguas excesiva en relación con la correspondiente a la situación de dichos tramos, que puede ser debida a condiciones naturales de las aguas en ciertas zonas o regiones (sustratos fuertemente calizos, altas temperaturas, etc.) o a la contaminación antropógena causada por vertidos órganicos de origen urbano, industrial, agrícola, etc.

- Especies indicadoras de contaminación: Son las que proliferan en las aguas más contaminadas donde existen condiciones de anoxia más o menos importantes, formando unas comunidades de macroinvertebrados muy simplificadas cuya diversidad es a veces mínima. En estas aguas, la vida se desarrolla fundamentalmente al nivel de los microorganismos, siendo muy abundantes en ellas las 
poblaciones de bacterias y hongos que aprovechan estas cantidades ingentes de materia orgánica.

Las especies indicadoras de contaminación pueden estar presentes en los tramos altos de los ríos donde las aguas no presentan déficits de oxígeno y, en este caso, se sitúan en las zonas de menor corriente, a veces en el medio hiporreico, formando poblaciones muy escasas.

$\mathrm{Su}$ abundancia excesiva en ciertos tramos de los ríos, ocupando las facies Iótica o de corriente es lo que refleja el grado de contaminación orgánica de las aguas, siendo en estos casos "fuertemente indicadoras" de dichas condiciones, al tener, con caracter estenoico, su preferencia ecológica en estos tramos de intensa contaminación.

El factor sinecológico del índice propuesto también es variable, en estos casos no con el tramo del río que estamos valorando sino con el aspecto cualitativo de la comunidad a la que se refiere.

El parámetro sinecológico más indicativo de la estructura de la comunidad es la "diversidad", calculada a traves de alguna de las fórmulas relaciondas con ella. Los valores de diversidad altos corresponden a unas comunidades complejas y estables, desarrolladas en medios donde existen numerosas interrelaciones físico-químicas y biológicas que autorregulan las poblaciones de cada especie. Los valores de la diversidad bajos se refieren a las comunidades que viven en unas condiciones extremas donde algunas especies, muy adaptadas a ellas, proliferan excesivamente con respecto a las restantes de la comunidad

La contaminación de las aguas supone la aparición de estas condiciones extremas en las que solo un número reducido de especies puede existir, a veces con poblaciones muy numerosas al disminuir o desaparecer por completo la competencia de las restantes que no toleran dichas condiciones.

I.a diversidad es un parámetro que está, por tanto, muy relacionado con la contaminación de las aguas, siendo más baja o mínima cuanto mayor sea ésta, aunque las condiciones extremas que causan algunos valores muy pequeños de la diversidad de las comunidades pueden ser debidas a causas naturales ajenas a la contaminación. (p. e. sustratos arenosos, fluctuaciones de caudal, etc.).

En el índice biológico propuesta la función del factor sinecológico se hace igual a la expresión de la diversidad de la comunidad, calculada con la fórmula de Shannon-Weaver, cuando el factor autoecológico es positivo (en los casos en que las especies intolerantes $\mathrm{o}$ indiferentes dominan sobre las restantes); y se hace igual a la inversa de dicha expresión cuando el factor autoecológico es negativo (en casos en que la comunidad está constituida fundamentalmente por especies características de aguas eutrofizadas o contaminadas).

De esta forma, el factor sinecológico o de diversidad potencia el resultado del índice cuando las comunidades están constituidas por especies de aguas "limpias", haciéndolo mayor cuanto mayor es la diversidad de las comunidades existentes. Y disminuye el valor negativo del índice en los casos en que, siendo más abundantes las especies de aguas contaminadas, existe una diversidad más alta que en otras zonas de peores condiciones del medio.

El índice propuesto, como se comprueba, toma valores positivos o negativos según la composición o "calidad" de las especies que forman la comunidad valorada, alcanzando valores más altos cuanto mayor es su diversidad.

Para la aplicación de este índice biológico a la cuenca del Duero hemos establecido una zonación en la misma, diferenciando tres zonas o regiones (Fig. 1), que integra los resultados físico-químicos $\mathrm{y}$ biológicos obtenidos en el presente estudio y responde a la zonación potencial de la cuenca según sus características naturales teóricas, de no existir ciertos factores limitantes, de acción local, o algunos focos puntuales de contaminación, que alteran las condiciones físico-químicas de las aguas y modifican de forma más o menos completa su estado actual.

Esta zonación teórica se ha representado en la Figura 1 y en ella se distinguen tres zonas o regiones:

-Zona I o de Rhithron: Corespondiente a las cabeceras de los ríos estudiados, exceptuando las de los que discurren por los valles de menor pendiente, y a los tramos medio-altos situados en las regiones de montaña.

- Zona II o de Epipotamon: Correspondiente a los tramos medios y bajos de los ríos estudiados, exceptuando los más caudalosos de la cuenca. En esta zona se consideran también las cabeceras de los ríos pequeños no incluidos en la Zona I.

- Zona III o de Mesopotamon: Está constituída por el tramo del río Duero aguas abajo de su cofluencia con el Pisuerga y por los tramos bajos del Pisuerga, Esla y Tormes, de mayor caudal en la cuenca.

El índice biológico se ha calculado a partir de las comunidades de macroinvertebrados de la facies Iótica 


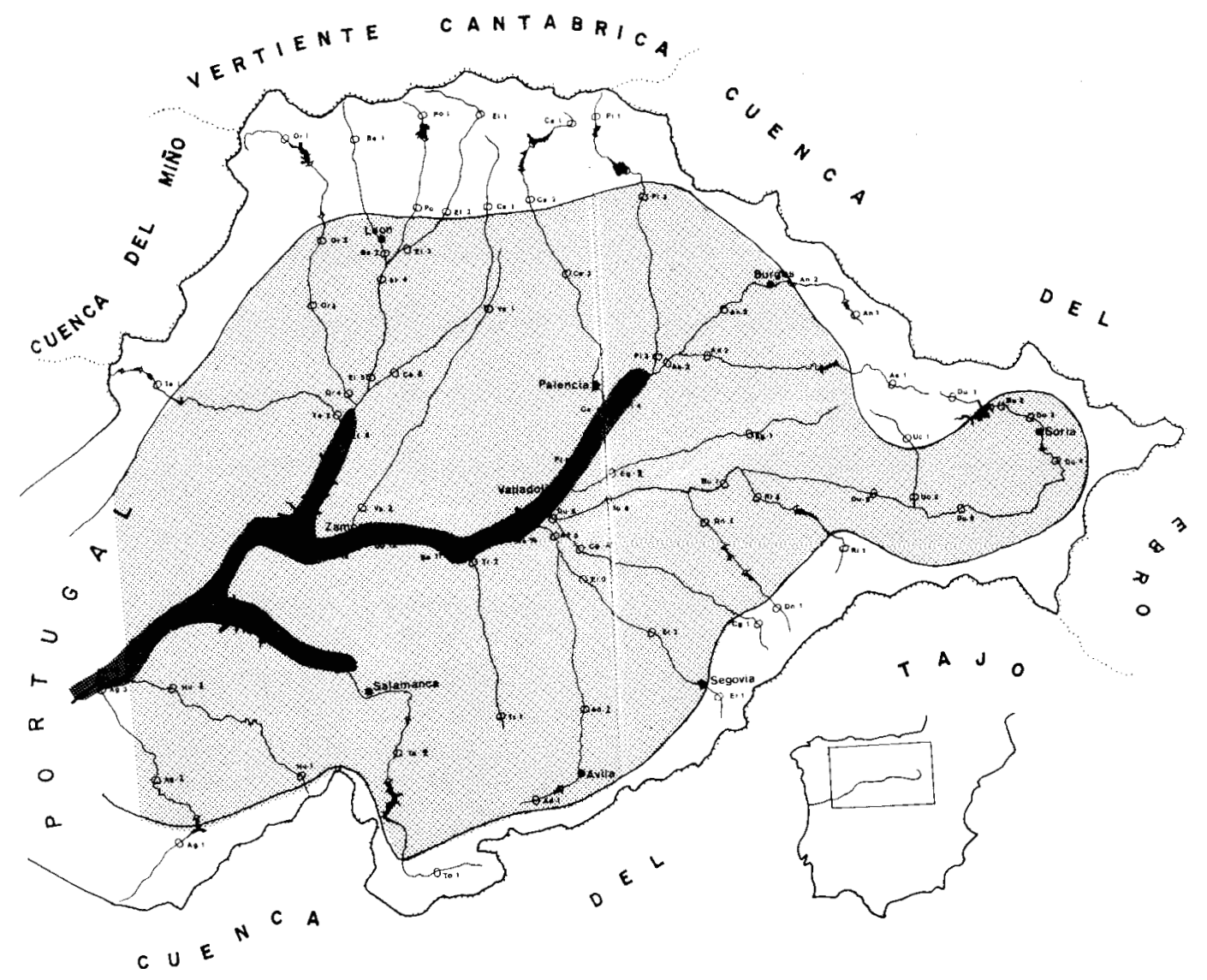

Figura 1.-Zonación teórica de la cuenca del Duero

Theretical zonation of the Duero Basin.

de los ríos, habiendo establecido previamente la siguiente relación de especies presentes en la cuenca del Duero:

\section{Especies intolerantes}

\section{Planariidae}

Baetis alpinus

Baetis melanonyx

Acentrella sinaica

\section{Epeorus torrentium}

Rhithrogena gr. diaphana

Rhithrogena gr. bybrida

Rbithrogena semicolorata

Serratella albai

Serratella hispanica
Drunella paradinasi

Eurylopbella iberica

Torleya mayor

Calliarcys bumilis

Habroleptoides umbratilis

Habrophlebia nervulosa

Thraulus bellus

Bracbyptera arcuata

Amphinemura sulcicollis

Protonemura hispanica

Protonemura intricata

Protonemura meyeri

Protonemura pyrenaica

Leuctra gr. hippopus

Leuctra gr. fusca

Isoperla sp. 
Dinocras cepbalotes

Perla bipunctata

Perla burmeisteriana

Perla grandis

Perla marginata

Siphonoperla torrentiem

Boyena irene

Cordulegaster annulatus

Dupopbilus brevis

Elmis aenea

Elmis maugetii

Elmis rioloides

Esolus angustatus

Limnius opacus

Limnius perrisi

Hydraena brachymera

Hydraena cordata

Hydraena corinna

Hydraena inapicipalpis

Haenydra emarginata

Haenydra exasperata

Haenydra iberica

Haenydra truncata

Helodidae

Rhyacophila dorsalis

Rbyacopbila lusitanica

Rhyacophila martynovi

Rhyacophila meridionalis

Rbyacophila mocsaryi

R byacophila obliterata

Rhyacophila occidentalis

Rhyacophila pulchra

Rhyacophila relicta

Rhyacophila terpsichore

Glossosomatidae

Philopotamus variegatus

Philopotamus montanus

Wormaldia variegata

Hydropsycbe pellucidula

Hydropsyche siltalai

Hydropsyche tibialis

Hydropsyche cf. dinarica

Polycentropus king $i$

Plectrocnemia sp.

Micrasemasp.

Limnephilidae

Silo graellsi

Larcasia partita

Thremma tellae

Lepidostoma birtum
Adicella reducta

Athripsodes albifrons

Athripsodes bilineatus

Sericostomatidae

Odontoceridae

Blephariceridae

Tabanidae

Athericidae

Dicranota sp.

Psychodidae

Prosimulium sp.

\section{Especies indiferentes}

Theodoxus sp.

Ancylus fluviatilis

Sphaeriidae

Lumbricidae

Glossiphonidae

Haemopis sanguisuga

Erpobdella sp.

Baetis muticus

Baetis rhodani

Centroptilum luteolum

Centroptilum pennulatum

Oligoneuriella rhenana

Ecdyonurus insignis

Ecdionurus lateralis

Ecdyonurus sp.

Epbemerella ignita

Paraleptophlebia cincta

Paraleptophlebia submarginata

Ephemera danica

Brachyptera braueri

Brachyptera vera

Taeniopteryx schoenemundi

Rhabdiopteryx thienemanni

Nemoura sp.

Euleuctra geniculata

Capniidae

Perlodes microcephala

Hemimelaena flaviventris

Odonata (Zygoptera)

Gomphidae

Heteroptera

Hydradephaga

Esolus paralelepipedus

Limnius wolckmari

Oulimnius troglodytes

Stenelmis canaliculata 
Sialidae

Rhyacophila denticulata

Rhyacophila munda

Hydroptilidae

Polycentropus flavomaculatus

Hydropsyche pictetorum

Psychomyia pusilla

Bracbycentrus subnubilus

Goera pilosa

Atbripsodes braueri

Leptocerus lusitanicus

Triaenodes ochrellus

Tipulidae

Hexatoma $s p$.

Antocha sp.

Dixidae

Metacnephia sp.

Odagmia sp.

Tetisimulium $s p$.

Simulium $s p$.

Tanypodinae

Diamesinae

Ortbocladinae

Tanitarsini

Ceratopogonidae

Stratiomyidae

Dolichopodidae

Limnopbora sp.

Especies de aguas eutróficas:

Pbysidae

Lymnaeidae

Planorbidae

Unionidae

Helobdella stagnalis

Atyaepbyra desmarestii

Astacidae

Gammaridae

Siphlonurus sp.

Baetis atrebatinus

Baetis buceratus

Baetis digitatus

Baetis fuscatus

Baetis gr. lutberi

Baetis vardarensis

Baetopus tenellus

Cloeon dipterum

Cloeon simile

Procloeon sp.
Oligoneuriella duerensis

Oligoneuriopsis skbounate

Heptagenia coerulans

Heptagenia sulphurea

Ephemerella mesoleuca

Caenidae

Choroterpes picteti

Leptopblebia marginata

Ephemera lineata

Potamantbus luteus

Ephoron virgo

Dryops sp.

Esolus pygmaeus

Limnius intermedias.

Oulimnius rivularis

Riolus cupreus

Riolus illiesi

Hydrophiloidea

Chimarra marginata

Hydropsyche bulbifera

Hydropsyche exocellata

Hydropsyche lobata

Cheumatopsyche lepida

Polycentropus telifer

Psychomyia ctenophora

Ecnomus deceptor

Mystacides azurea

Setodes argentipunctellus

Ceraclea dissimilis

Anopbeles sp.

Eusimulium sp.

Wilhelmia sp.

Obuchovia sp.

Chironomini

Empididae

Especies características de contaminación

Tubzfzczdae

Chironomus gr.plumosus

Cbironomus gr. thummi

Syrpbidae

Para cada estación de muestreo se ha considerado un valor de diversidad medio (media de las diversidades obtenidas en cada periodo de muestreo) y su composición faunística a lo largo del año (incluyendo todas las especies que en una época o en otra están presentes en la estación), habiendo obtenido los valores expuestos en la Fig. 3. 
Los valores más altos del índice se producen en las cabeceras de los ríos Duero, Arlazón, Carrión, Esla, Porma, Orbigo, Tera, Riaza, Eresma, y Tormes, alcanzando el máximo absoluto en el río Carrión donde existen unas comunidades muy diversas, constituidas por un porcentaje muy elevado de especies intolerantes a la contaminación

Los valores más bajos del índice corresponden a las estaciones más contaminadas de la Cuenca, como son la estación del Duero en Villamarciel, la del Pisuerga en Simancas, la del Arlazón en Villodrigo y la del Bernesga en Alija, en las que existen unas comunidades de macroinvertebrados muy simplificadas, constituidas prácticamente en su totalidas por especies indicadoras de contaminación.

Con el fin de resaltar mejor las variaciones de calidad y poder representar gráficamente los valores del índice biológico en los distintos tramos de los ríos de la Cuenca del Duero, hemos estrablecido las siguientes clases, reflejadas en la Fig. 2:

$\begin{array}{lrl}\text { Clase I } & 100 & <\mathrm{Ib} \\ \text { Clase II } & 50<\mathrm{Ib} & <100 \\ \text { Clase III } & 10<\mathrm{Ib}<50 \\ \text { Clase IV } & 0<\mathrm{Ib}<10 \\ \text { Clase V } & -10<\mathrm{Ib}<0 \\ \text { Clase VI } & -50<\mathrm{Ib}<-10 \\ \text { Clase VII } & & \mathrm{Ib}<-50\end{array}$

El estado actual de los ríos de la Cuenca del Duero, estimado a través del índice biológico propuesto, es un punto de referencia para conocer la evolución futura de las aguas según su recuperación o progresiva degradación biológica.

Este índice constituye, por tanto, un sistema de control de la calidad de las aguas que deberia ser utilizado de forma periódica como complemento a la información físico-química obtenida por medio de los análisis de aguas tradicionales, los cuales en ocasiones no reflejan de forma real el estado o grado de alteración del medio acuático.

\section{DISCUSION}

Para comprobar la validez del método empleado hemos calculado, con los datos del río Duero, otros índices comunmente utilizados en los trabajos de calidad de agua. Así, en la Fig. 3 se han representado los valores del índice de Tuffery yVernaux (1976), como media aritmética de los cuatro valores del índice correspondientes a cada uno de los periodos del año en que se efectuaron los muestreos; los del índice de diversidad, según la fórmula de ShannonWeaver (1963) y los del índice de calidad general (Mingo, 1982) atendiendo a ciertos parámetros físicos químicos de las aguas. El índice biológico diseñado para la Cuenca del Duero se expresa en valores absolutos y en valores discretos según la clase a la que pertenece cada estación de muestreo.

Se observa que, en el río Duero, el índice físicoquimico tiene mucha menos sensibilidad que los restantes biológicos. Respecto a éstos, todos ellos reflejan un mínimo absoluto en la estación 10, aguas abajo de la llegada del río Pisuerga al Duero, y dos mínímos relativos, uno en la estación 4, aguas abajo de la ciudad de Soria, y otro en la estación 12. El de esta última hay que interpretarlo teniendo en cuenta que corresponde a un tramo de recuperación del Duero despúes de recibir al Pisuerga, que abarca desde la estación 10 hasta la 13 , a partir de la cual el río Duero forma una serie continuada de embalses donde sufre una auténtica autodepuración de sus aguas; yque un valor más alto del índice en la estación 11 se debe únicamente a que esta estación se localizóen un tramo sobreoxigenado, inmediatamente aguas abajo de la presa de Castronuño

$\times$ De forma general puede decirse que el río Duero lleva en su parte alta unas aguas de buena calidad; sufre un deterioro a su paso por Soria; después se recupera ligeramente $\mathrm{y}$, hacia aguas abajo, va perdiendo calidad biológica gradualmente para llegar a su tramo más contaminado, en las proximidades de Valladolid, donde recibe al río Pisuerga, de mayor caudal que el Duero en su confluencia y apreciablemente contaminado después de recoger los vertidos de Palencia, a través del río Carrión, y los de Valladolid. Posteriormente inicia su recuperación de forma progresiva, no viéndose apenas afectado por los vertidos de Zamora, a excepción de una pequeña zona antes de "Los Arribes", donde confluyen las aguas de los ríos Esla y Tormes, para llegar a la frontera de Portugal con una calidad de sus aguas, tanto físico-química como biológica, equivalente a la de algunos tramos de su parte alta.

Esta evolución general de la calidad de las aguas del Río Duero la recogen todos los índices biológicos, si bien el de Tuffery y Verneaux marca un máximo absoluto en la estación 6, en San Esteban de Gormaz, donde, aunque existen varias especies de plecópteros 


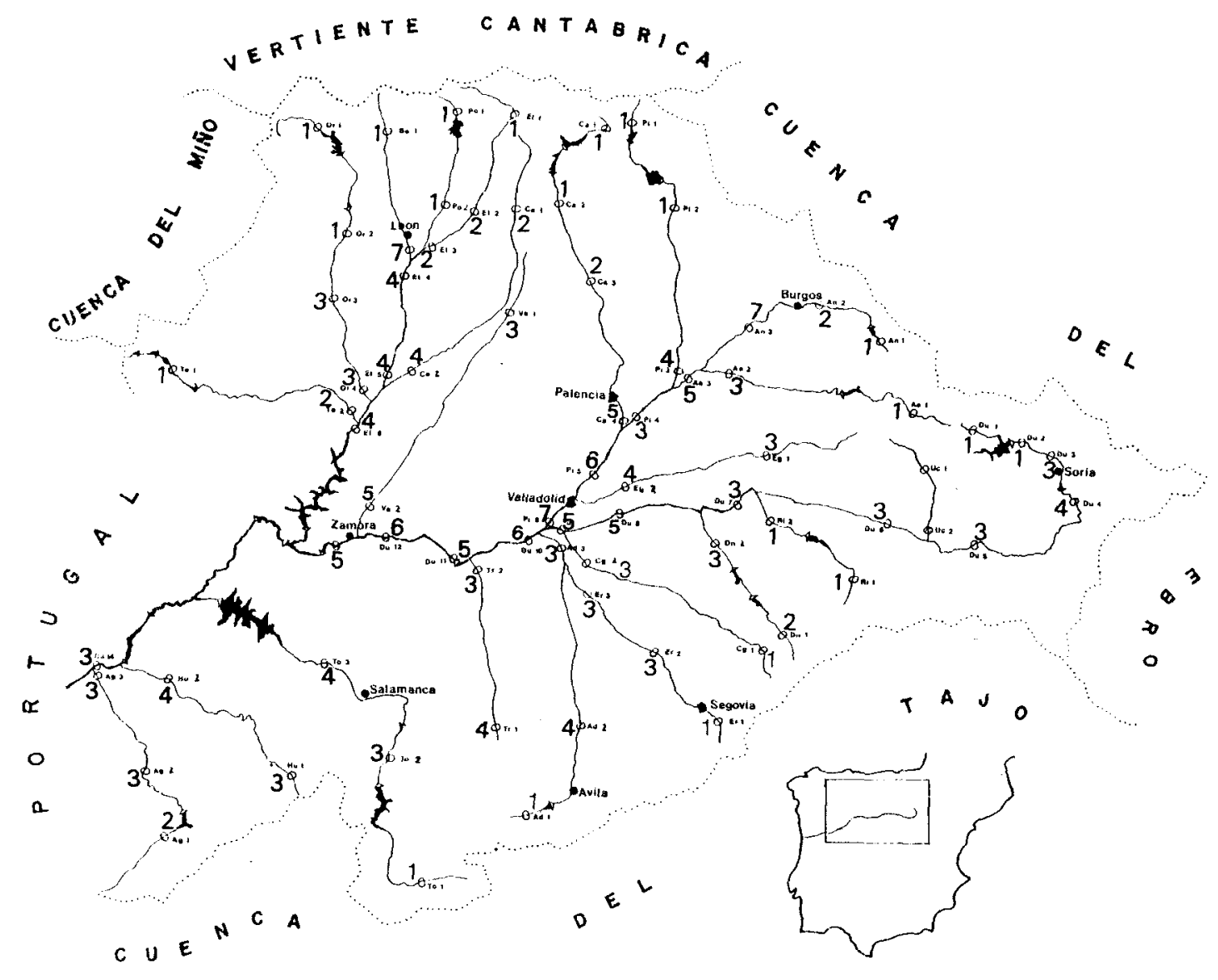

Figura 2.- Valor del nuevo índice biológico en los distintos punros de muestreo de la cuenca del Ducro

Values of the new biological index in the localities of the Duero Basin.

que motivan un valor alto de este índice, la gran mayoría de la comunidad de macroinvertebrados corresponde a poblaciones de especies de carácter indiferente, o incluso indicadoras de eutrofización, y las aguas se encuentran en un estado mucho más deteriorado, como resultado evidente, que en las estaciones de cabecera del río. Por otra parte, este índice no refleja la recuperación de las aguas que llegan a la estación 14 ya que en ellas no existen especies "indicadoras de calidad" (plecópteros) pero tampoco existen "indicadores de contaminación", y las comunidades de macroinvertebrados están muy reducidas, no a causa del factor "calidad del agua" sino,por el contrario, a causa de la inestabilidad del sustrato y del caudal, como consecuencia de la regulación de los embalses de aguas arriba para producción de energía hidroeléctrica

Respecto al índice de diversidad se observa que ésta sigue más fielmente la evolución de calidad de las aguas del río Duero que el de Tuffery y Vernaux, aunque se muestra poco sensible ya que sus valores oscilan dentrode un intervalo relativamente pequeño. Así mismo, hay que resaltar que en la estación 10 , si bien la comunidad de macroinvertebrados está muy simplificada, el valor de su diversidad es relativamente alto al no haber ninguna especie en franca dominancia respecto a las restantes, efecto por otra parte esperado al existir en ese tramo una contaminación de tipo industrial más que orgánico (Verneaux, 1976), y el indice no refleja claramente la gran diferencia que existe entre la calidad de las aguas en este tramo de río y en los restantes. 


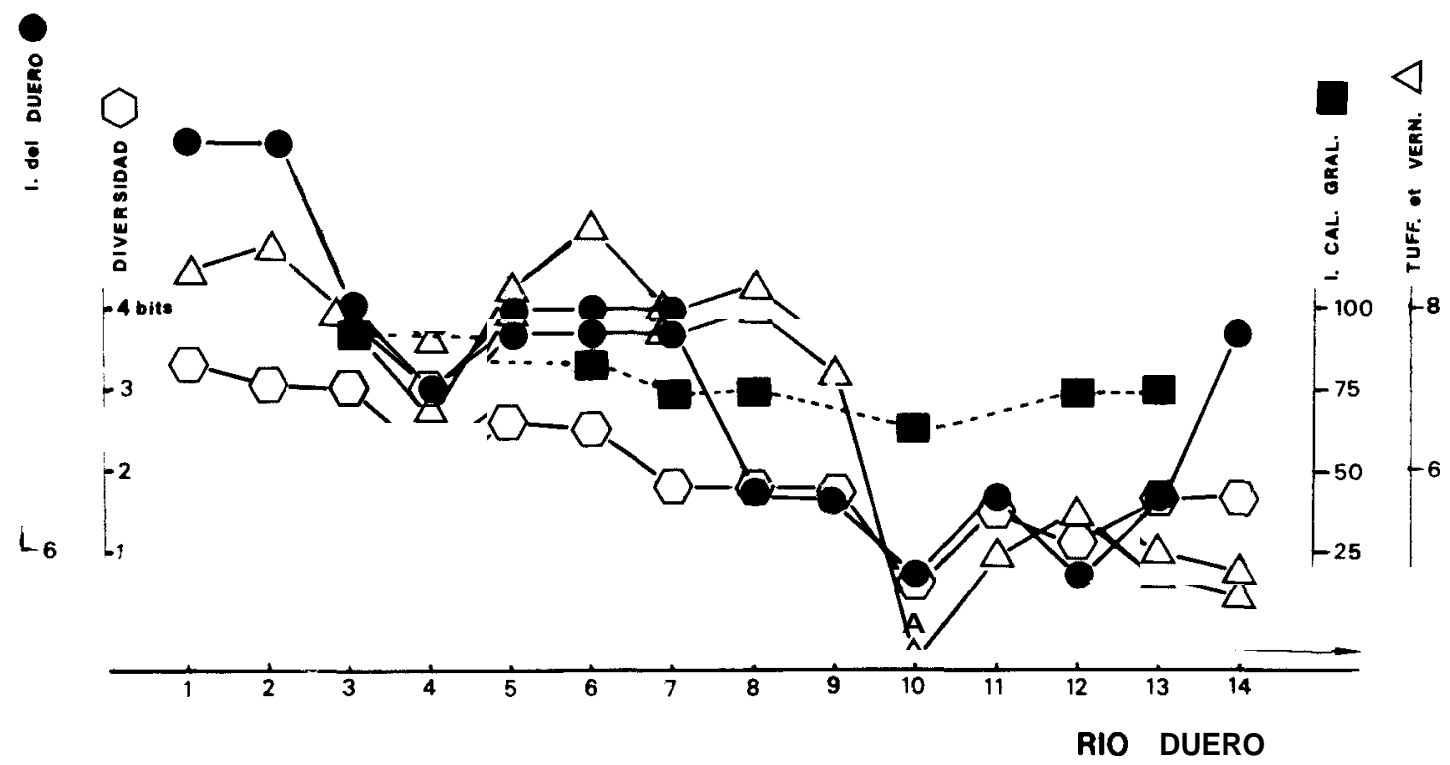

Figura 3 - Comparación de los valores de distintos indices (índice de calidad general; indice de Tuffery y Verneaux; indice de diversidad; nuevo índice) a lo largo del curso del río Duero.

Comparison of different indices (general quality index; Tuffery \& Verneaux; diversity index; new index) along the River Duero.

Finalmente, el índice diseñado para la Cuenca del Duero también recoge estas variaciones de calidad de las aguas demostrando que dicho índice, expresado en clases, es el que mejor se ajusta a la variación del factor "calidad del agua", estimado a traves de las comunidades de macroinvertebrados presentes en el río, sin que en él intervengan otros factores ajenas al mismo que puedan distorsionar o hacer difícil la interpretación de los resultados obtenidos.

\section{BIBLIOGRAFIA}

Balloch, D.; Davies, C.E. \& Jones, F.H. 1976. Biological assessment of water quality in three British river. Wat. Pollut. Control, Lond. 75: 92-110.

Beck, W.M. 1955. Suggested method for reporting biotic data. Sewage and Ind. Wastes 27(10): 1193-1197.

García de Jalón, D. y González del Tanago, M . En prensa Estimación de la contaminación de las aguas mediante indicadores biológicos: Aplicación al ríoJarama. Dip. Provincial de Madrid Publ Ghetti, P. F. y Bonazzi, G., 1977. A comparison between vatious criteria for the interpretation of biological data in the analysis of the quality of running waters. Water Research II: 819-831.

González del Tanago y García de Jalón, D. En prensa. Estudio para una metodología de clasificación biotipológica de los ríos de la Cuenca del Dnero. Ceotma, Mopu, Madrid.
González del Tanago, M., García de Jalón y Martínez Elcoro, I. 1979. Estudio de la fauna macroinvertebrada de los ríos Ciguela, Záncara y Córcoles: Aplicación de índices biológicos para el estudio de la calidad de sus aguas. Bol. Est. centr. Ecol. 15: 45-49.

Hawkes, H. A., 1978. Conceptual basis for the biological surveillance of river water quality. En : Biological Surveillance of River Water Quality. Procs. Section K \& Section J. British Assoc. Advancement of Science. Aston, Birmngham.

Hellawell, J. M., 1978. Biological Surveillance of Rivers. Water Research Centre Publ. Stevenage, Inglaterra.

Jensen, F. y Aagaard, P., 1981. A critical comparison of some indices used for measuring stream pollution. Natura Jutlandica 19: 147-160.

Kolkwitz, R. y Marsson, M. 1902. Grundsatze fur die biologische Beurteilung des Wassers nach seiner Flora und Fauna. Mitt. a. d. Kgl. Prufungsanst. f. Wasserversorg. u. Abwasserbeseitigung zu Berlin 1: 33-72.

-1908. Okologie der pflanzlichen Saprobien. Ber Dt. Botan. Ges. 261: 505-519.

-1909. Okologie der tierischen Saprobien. Int. Rev. ges. Hydrobiol 2: 125-152.

Margalef, R. 1951. Diversidad de especies en las comunidades naturales. Pub. Inst. Biol. apl. Barcelona 6: 59-72.

Menhinick, E. F. 1964. A comparison of some species-individuals diversity indices applied to samples of field insects. Ecology 45: 859-861.

Shannon, C.E. y Weaver, W. 1963. The mathematical theory of Comunication. Univ. of Illinois Press. 117 pp.

Simpson, E.H., 1949. Measurement of diversity. Nature Lond. 
163: 688 .

Tuffery, G. y Verneaux, J. 1967. Méthodes de determination de la qualité biologique des eaux courantes. Trav. Sect. Tech. P. et P. Paris.

Verneaux, J., 1976. Application de la méthode des "indices biotiques" à l'échelle d'un reseau hydrographyque-cartographie de la qualité biologique des eaux. p. 221-285. En: La pollution des eaux continentales. Incidence sur les biocénoses aquatiques. Ed. P. Pesson. Gauthier-Villars.

Wihm, J. 1975. Biological indicators of pollution En: River Ecology B. A. Whitton Ed. Blackwell Scientific Publ. p. 375-402. Woodiwiss, F. S. 1964. The biological system of stream classification used by the Trent River Board. Chemistry \& Industry 11: 443-447 